\title{
Processo de enfermagem em pacientes submetidos à angioplastia transluminal percutânea coronária
}

\author{
Nursing process in patients submitted to coronary percutaneous transluminary angioplasty \\ Proceso de enfermería en pacientes sometidos a angioplastia coronaria transluminal \\ percutánea
}

Marcos Gabriel de Jesus Rodrigues ${ }^{1 *}$, Reinaldo da Silva ${ }^{1}$, Mirielle Dias Gonçalves ${ }^{1}$, Alanna Fernandes Paraíso'.

\section{RESUMO}

Objetivo: O presente estudo teve como objetivo descrever a assistência de enfermagem em pacientes submetidos à angioplastia transluminal percutânea coronária por serviços de hemodinâmica na cidade de Montes Claros, norte de Minas Gerais, Brasil. Métodos: Constitui-se em estudo qualitativo conduzido com 10 (dez) profissionais da equipe de enfermagem atuantes em dois serviços de hemodinâmica hospitalar de Montes Claros-MG. A coleta de dados foi realizada por entrevistas contendo um roteiro de perguntas estruturadas. As falas foram gravadas, transcritas na íntegra e os achados analisados pela técnica de análise do discurso. Resultados: Os enfermeiros apontaram as dificuldades no controle de materiais passíveis de reuso, relação interpessoal com a equipe, também apontam satisfação da experiência adquirida no serviço de hemodinâmica. A equipe de enfermagem explanou sobre a importância e a necessidade do processo de enfermagem adequado para o paciente. Conclusão: Após análise, possibilitou-se a descrição do processo de enfermagem aplicado no pré, trans e pós-procedimento. Também, verificou-se a importância de um processo de enfermagem adequado e eficaz para o paciente do ambiente hospitalar e após a alta.

Palavras-Chave: Angioplastia, Assistência, Enfermagem, Hemodinâmica.

\begin{abstract}
Objective: The present study was intended to describe the nursing assistance in patients subjected to percutaneous coronary angioplasty by hemodynamic services in the city of Clear Hills, north of Minas Gerais, Brazil. Methods: It is a qualitative study conducted with 10 (TEN) professionals of the nursing team acting in two services of hospital hemodynamic of Clear Hills-MG. The data collection was carried out by interviews containing a roadmap of structured questions. The lines were recorded, transcribed in full and the findings analyzed by the technique of analysis of the speech. Results: Nurses pointed out the difficulties in the control of reused materials, interpersonal relationship with the team, also indicate satisfaction of the experience acquired in the Hemodynamic service. The nursing staff has explaned on the importance and necessity of the nursing process suitable for the patient. Conclusions: After analysis, the description of the nursing process applied in the pre, trans and go was made possible. Also, it was verified the importance of an adequate and effective nursing process for the patient of the hospital environment and after the discharge.
\end{abstract}

Key words: Angioplasty, Assistance, Nursing, Hemodynamic.

${ }^{1}$ Faculdade de Saúde e Desenvolvimento Humano Santo Agostinho, Montes Claros - MG.

*E-mail: librasmarcos@gmail.com

SUBMETIDO EM: 2/2019

ACEITO EM: 3/2019

PUBLICADO EM: 5/2019

REAS/EJCH | Vol.Sup.23 | e284 | DOI: https://doi.org/10.25248/reas.e284.2019 Página 1 de 9 


\section{RESUMEN}

Objetivo: El presente estudio tuvo como objetivo describir los cuidados de enfermería en pacientes sometidos a angioplastia coronaria transluminal percutánea por los servicios de hemodinámicos en la ciudad de Montes Claros, Minas Gerais, Brasil. Métodos: Constituye el estudio cualitativo realizado con 10 (diez) de enfermería equipo de profesionales activos en dos servicios hemodinámicas de Montes ClarosMG. Los datos fueron recogidos a través de entrevistas con un guión de preguntas estructuradas. Se registraron las líneas transcritas en full y los resultados analizados mediante la técnica de análisis del discurso. Resultados: Las enfermeras indicadas las dificultades en el control de los materiales que pueden reutilizarse, relación interpersonal con el equipo, también indican satisfacción de experiencia de servicio hemodinámica. El equipo de enfermería, explicó sobre la importancia y necesidad del proceso de enfermería adecuado para el paciente. Conclusiones: Después de análisis, permitió-en caso la descripción del proceso de enfermería aplicado en el pre, trans y post atención. También, fue la importancia de un proceso adecuado y eficaz de enfermería al paciente en el hospital y después de la descarga.

Palabras clave: Angioplastia, Asistencia, De enfermería, Hemodinamia.

\section{INTRODUÇÃO}

As doenças cardiovasculares (DCV) são um grupo de diversas doenças do coração e dos vasos sanguíneos que geralmente são causadas por algum fator que dificulte a circulação sanguínea efetiva (Brasil, 2016). Em virtude disso, dados apontaram que 17,7 milhões das pessoas morreram por doenças cardiovasculares em 2015, totalizando 31\% em nível global. Além disso, no mesmo ano, estima-se que 7,4 milhões de óbitos ocorreram por doenças coronarianas (BRASIL, 2017).

Dentre os diversos tipos de patologias cardiovasculares destaca-se a aterosclerose, alteração causada por acúmulo de placas de gordura que se alojam em diversas regiões arteriais, dificultando o fluxo sanguíneo. Assim que ocorre obstrução total do vaso, inicia-se o processo de morte celular do miocárdio configurando em Infarto Agudo do Miocárdio (IAM), condição grave e que necessita de atendimento especializado e imediato (OLIVEIRA MF e SILVA LF, 2003).

Segundo Barbosa MHM et al. (2013), como forma de intervenção para o IAM, observaram-se crescimentos de técnicas percutâneas de revascularização nos últimos anos. O aperfeiçoamento do instrumento de angioplastia transluminal percutânea coronária e o aparecimento de novos dispositivos, como stents tem possibilitado o tratamento da maioria dos pacientes. A angioplastia é considerada a terapêutica preferencial para tratamento e profilaxia do IAM, procedimento que permite a reperfusão coronariana, principalmente nas primeiras doze horas (TAETS GGC, 2016).

Para assistir esse paciente como um todo, o enfermeiro poderá utilizar de ferramentas que permitem desenvolver uma prática assistencial com as melhores evidências científicas e com o respaldo ético-legal. Assim, o Processo de Enfermagem é uma metodologia de organização, planejamento e execução das ações de enfermagem que são realizadas pela equipe durante o período em que o indivíduo se encontra sob a assistência de enfermagem de forma sistematizada (SANTOS WN,2014). Dividido nas etapas: Histórico de Enfermagem ou Avaliação Inicial, Diagnóstico de Enfermagem, Planejamento, Implementação e Evolução de Enfermagem (DELLíACQUA MCQ e MIYADAHIRA AMK, 2002), o processo de enfermagem oferece inúmeros subsídios para o desenvolvimento de metodologias interdisciplinares e humanizadas de cuidado. Sua aplicação nas instituições seja de atenção primária ou terciária apresenta muitos benefícios como segurança no planejamento, execução e avaliação das práticas assistenciais de enfermagem, cuidado centrado no indivíduo, autonomia ao profissional enfermeiro, diminuição do tempo de tratamento e consequente economia de insumos e recursos (SANTOS WN, 2014). 
Conhecer o processo de enfermagem proporciona a aplicação efetiva de todas as suas etapas, conduzindo à melhoria da qualidade da assistência além de agregar na estruturação de um saber teórico para melhorar a prática clínica utilizada pelo enfermeiro em cardiologia (TAETS GGC, 2016).

Considerando que o PE desenvolvido adequadamente reduzirá as complicações atuais ou futuras para os pacientes submetidos à angioplastia coronária - já que é um instrumento de trabalho fundamental para o enfermeiro - esse estudo objetivou descrever a assistência de enfermagem em pacientes submetidos à angioplastia transluminal percutânea coronária no período pré, trans e pós-procedimento por serviços de hemodinâmica na cidade de Montes Claros, norte de Minas Gerais, Brasil.

\section{MÉTODOS}

Consiste de estudo descritivo, de abordagem qualitativa. Os sujeitos do estudo foram 02 profissionais enfermeiros e 08 técnicos de enfermagem que atuam nos serviços de Hemodinâmica do Hospital Irmandade Nossa Senhora das Mercês, instituição filantrópica, beneficente, referência para o norte de Minas Gerais e sul da Bahia, certificado pela Organização Nacional de Acreditação (ONA) e Ministério da Saúde em Hospital Acreditado com Excelência. Atualmente conta com 392 leitos, sendo 80\% deles destinados ao Sistema Único de Saúde (SUS). Fundação Dilson Godinho de Quadros de Montes Claros é uma entidade civil, filantrópica e beneficente. Tornou-se referência na assistência integral à saúde nas áreas de oncologia e nefrologia e oferece também serviços de alta complexidade nas mais diversas especializações médicas, possuindo 117 leitos instalados, sendo $77 \%$ destinados ao SUS. Inclui-se no estudo profissionais que expressaram seu desejo em participar voluntariamente da pesquisa, através da assinatura do Termo de Consentimento Livre e Esclarecido.

Os participantes foram submetidos a um roteiro de entrevista semiestruturado contendo 12 e 10 questões para o enfermeiro e técnico de enfermagem, respectivamente, norteando-os na abrangência do papel da equipe de enfermagem no serviço de hemodinâmica - vivências e dificuldades, processo de enfermagem no pré, trans e pós-procedimento de angioplastia transluminal percutânea coronária, e orientações para alta hospitalar. Variáveis sobre o perfil dos profissionais foram coletadas para caracterização dos sujeitos do estudo. O questionário foi elaborado pelos pesquisadores de acordo com o objetivo proposto. As entrevistas foram realizadas no período de abril e maio de 2018.

Os participantes foram identificados através de siglas, enfermeiros (Enf) e técnicos de enfermagem (Tec.Enf) seguindo o número de questionário. Os dados foram coletados por instrumento de gravador e transcrito na íntegra. As falas foram analisadas pela técnica de análise de discurso.

Com o objetivo de atender às Diretrizes e Normas Regulamentadoras do Conselho Nacional de Saúde através da Resolução n 466 de 2012, foi submetido ao Comitê de Ética em Pesquisa das Faculdades Integradas Pitágoras de Montes Claros e autorizado através do parecer de número 2.577.915/2018.

\section{RESULTADOS E DISCUSSÃO}

A amostra foi de 10 membros da equipe de enfermagem sendo eles: 02 enfermeiros, com especialização em cardiologia ou urgência e emergência, e 08 técnicos de enfermagem, sendo apenas 1 com curso de aperfeiçoamento na área. As idades variaram de 24 anos a 50 anos, sendo 5 mulheres e 5 homens.

A análise das respostas obtidas pela equipe de enfermagem possibilitou a construção das seguintes categorias: (1) papel da equipe de enfermagem no serviço de hemodinâmica - vivências e dificuldades, (2) processo de enfermagem no pré, trans e pós-procedimento de angioplastia transluminal percutânea coronária e (3) orientações para alta hospitalar.

\section{Papel da equipe de enfermagem no serviço de hemodinâmica - vivências e dificuldades}

O enfermeiro é um profissional de extrema importância para atuar no serviço de hemodinâmica, pois desenvolve papéis fundamentais na gestão e na assistência (LEMOS IMN et al., 2017). Nesse contexto, o enfermeiro, ao desenvolver funções de liderança, também trabalha no gerenciamento de recursos humanos 
e materiais em serviços de hemodinâmica, o que exige tomada de decisões rápidas e precisas (LINCH GFC et al., 2010). Como afirmam os enfermeiros entrevistados.

- Enf01: (...) "O serviço de hemodinâmica é um serviço complexo que demanda gestão de pessoas, controle de materiais, manutenções, tem a questão também do agendamento dos procedimentos".

- Enf02: (...) "Eu considero minha experiência gerencial é excelente sabe, porque desde quando eu entrei na hemodinâmica a gente teve alguns processos que eram falhos, e atualmente a gente conseguiu evoluir muito".

Estudo publicado por Lemos IMN et al. (2017) mostra que por se tratar de um setor específico para exames por imagem, como cineangiocoronariográfica, arteriografia cerebral e de membros, angioplastia coronária, embolização de aneurisma, o serviço de hemodinâmica utiliza materiais diferenciados, como por exemplo: cateteres, introdutores, fios guias, contrastes. Estes materiais possuem um custo elevado, exclusivos e passíveis de reuso. Há uma grande necessidade de manutenção do estoque, justificando a necessidade de processos adequados para a compra deste. Portanto, o enfermeiro participa diretamente, na maioria das vezes, desses processos de aquisição ou requisição de materiais utilizados nesse serviço. Manter esses estoques adequados e atualizados pode ser um grande desafio para o enfermeiro ou profissional responsável.

- Tec.Enf01: (...) "No momento é a questão de material, geralmente o médico pede um material que não tem e aí acaba dificultando a realização de alguns exames".

- Enf01: (...) "As dificuldades que eu enfrento é em relação ao controle dos materiais pra gente conseguir manter a rastreabilidade".

-Tec.Enf02: (...) "As maiores dificuldades têm sido sobre material, material novo, material processado, a dificuldade de conseguir manter um estoque para poder atender o paciente de imediato, isso vai dificultando ao atendimento ao paciente".

No âmbito da liderança de equipe, são apontadas diversas dificuldades encontradas pelos enfermeiros, as quais se referem ao relacionamento com a equipe e as atribuições desses profissionais (AMESTOY SC et al., 2009). Os enfermeiros participantes do estudo apontam que a gestão de pessoas e o relacionamento interpessoal são fatores dificultadores e desafiadores na equipe de enfermagem atuante em serviços de hemodinâmica.

- Enf01: (...) "Também a questão da equipe, porque é uma equipe diferenciada que tem que ter conhecimentos, NE, na área".

- Enf02: (...) "E se a gente for falar outra dificuldade que a gente tem, eu acredito que não é só aqui, é a dificuldade com relacionamento interpessoal entre os profissionais, né, acho que já é uma característica de todos os setores fechados, né, a gente tem, sim, uma dificuldade em relação a isso".

Percebe-se que os cursos de graduação em Enfermagem, em sua maioria, não possuem em sua grade curricular conhecimentos profundos sobre cardiologia e radiologia. Porém, para que ocorra um funcionamento adequado do serviço de hemodinâmica, o enfermeiro precisa ter conhecimentos básicos sobre essas especialidades (VIEIRA LC et al, 2009).

-Enf01: (...) “É uma experiência que a gente não adquire na faculdade que vê muito pouco, né, sobre o serviço de hemodinâmica no geral".

Assim, o enfermeiro desenvolve suas atividades de forma generalista frente a uma equipe multidisciplinar. No entanto, para que consiga êxito em sua liderança, dependerá da forma como conseguirá influenciar as pessoas para que desempenhem suas atividades para a consecução de um objetivo comum. 


\section{Processo de enfermagem no pré, trans e pós-procedimento de angioplastia transluminal percutânea coronária}

O processo de enfermagem é composto por etapas, tais como históricos, anamnese (coleta de dados do cliente), diagnóstico, planejamento e prescrição dos cuidados de enfermagem, avaliações dos resultados obtidos (LEMOS IMN et al., 2017)

A aplicação do PE é ininterrupta e interligada, estratégia importante para a segurança do paciente. No pré-operatório, ocorre a visita de enfermagem para avaliar e preparar o paciente para o procedimento, buscando diminuir a possibilidade de eventos adversos (Monteiro EL et al., 2014).

-Enf01: (...) "No pré, nós temos a consulta de enfermagem que a gente verifica todo o preparo do paciente, quanto às medicações, é a suspensão da metformina, do marevan, se caso o paciente faz uso, a questão do jejum explicamos para o paciente todo o processo que é feito aqui no procedimento, é que é importante ele saber, até para tranquilizá-lo".

De acordo com Silva MLC (2017) a consulta de enfermagem contribuirá de forma esclarecedora, aliviando a ansiedade, respondendo às dúvidas do cliente acerca de todo o procedimento, contribuindo, também, para a avaliação de exames, preparo psicológico, orientações quanto ao preparo pré-operatório. Isso torna este processo menos traumático e doloroso.

Diante do exposto, a equipe de enfermagem aponta os itens da entrevista de enfermagem: Queixa Principal (QP), História Pregressa (HP), História Medicamentosa (HP), História Familiar (HF).

\section{Queixa Principal:}

- Enf02: (...) "O que o paciente está queixando no momento".

\section{História Pregressa:}

- Enf02: (...) "Possui comorbidades (Hipertensão, Diabetes Mellitus, entre outras"). (...) "Realizou alguma cirurgia (Qual?)".

- Tec.Enf03: (...) "Alergia a algum medicamento, alimento (peixe, frutos do mar), lodo.".

- Enf02: (...) "Etilismo. Tabagismo. Internações anteriores".

\section{História Medicamentosa:}

- Tec.Enf04: (...) "Quais medicamentos faz uso. Está fazendo uso de AAS, Clopidogrel ou Brilinta para o procedimento".

- Enf01: (...) "Suspendeu metformina / marevan e seus derivados por no mínimo três dias antes devido o uso do contraste".

- Tec.Enf03: (...) "Dados Vitais: Pressão Arterial. Frequência Cardíaca. Frequência Respiratória. Saturação de oxigênio".

- Tec.Enf05: (...) "Realizar punção venosa”.

- Enf01: (...) "Orientações: Verifica se realizou o jejum. Sobre o procedimento. Como se portar dentro da sala".

As atividades realizadas pela equipe de enfermagem durante a visita pré-operatória com foco na anamnese, exame físico e avaliação holística, contribuem para o levantamento dos dados do paciente e para um adequado preparo pré-operatório (MARCEDO VL, 2016). Em relação às etapas do processo de enfermagem para $\circ$ paciente que realizará $\circ$ procedimento de angioplastia transluminal percutânea coronária, após uma coleta efetiva e completa de dados, é possível detectar os seguintes diagnósticos de enfermagem, conforme abaixo descrito pelo Enf01. 
(...) "Alteração da pressão arterial, relacionado à ansiedade quanto ao procedimento, complexidade do procedimento".

(...) "Alteração do padrão de sono".

(...) "Débito cardíaco diminuído, relacionado à condição clínica do paciente se ele tiver um choque cardiogênico, lesões graves".

(...) "Mobilidade física prejudicada, relacionado ao repouso que o paciente faz após o procedimento".

(...) "Déficit no autocuidado, relacionado à internação e ao tratamento".

(...) "Dor aguda, relacionado à punção e também ao tratamento da lesão".

(...) "Eliminação urinária alterada, relacionado à ansiedade e contraste".

(...) "Integridade da pele prejudicada, relacionado ao dispositivo que é o introdutor".

(...) "Náuseas e vômitos, relacionado também ao uso do contraste e da ansiedade".

Estudo realizado sobre os principais diagnósticos de enfermagem de pacientes submetidos à angioplastia transluminal percutânea coronária pôde destacar: mobilidade física prejudicada, integridade da pele prejudicada, déficit no autocuidado, risco de lesão renal, dor aguda e débito cardíaco diminuído (LIMA LR et al., 2008).

Os diagnósticos de enfermagem aplicam o julgamento clínico das respostas do indivíduo, da família, dos sinais vitais, dos problemas de saúde atuais ou potenciais, fortalecendo a seleção das intervenções de enfermagem para alcançar resultados pelos quais o enfermeiro é responsável. A aplicação efetiva do processo de enfermagem conduz à melhoria da qualidade da assistência (TAETS GGC, 2016).

A equipe de enfermagem enumerou em relação aos procedimentos e condutas no período trans procedimento, os itens abaixo:

- Enf02: (...) "Realiza chek list verificando se os equipamentos estão funcionando, sendo eles: monitor multiparâmetros, desfibrilador, mesa de procedimento" (...) "Verifica se possui todos os materiais necessários para a realização do procedimento".

-Tec.Enf04: (...) "Recebe o paciente na sala de procedimento". Monitoriza para verificação de dados vitais (Pressão arterial, frequência cardíaca, saturação de oxigênio). Certifica novamente se possui alergia".

-Tec.Enf03: (...) "Realizar tricotomia. Realizar degermação. Montar a mesa estéril para o procedimento".

-Tec.Enf01: (...) "Administrar medicamento caso haja necessidade e de acordo com solicitação médica".

Mediante isso, o transoperatório é o período em que acontece a cirurgia propriamente dita e aplica na prática as ações planejadas conforme a prescrição de enfermagem (MONTEIRO EL et al., 2014).

Após o procedimento, o enfermeiro e técnico de enfermagem necessita realizar cuidados importantes com o paciente.

- Enf02: (...) "Retirar o introdutor".

- Tec.Enf01: (...) "Realizar uma compressão eficaz até obter hemostasia”.

- Tec.Enf02: (...) "Realiza o curativo compressivo de forma eficaz também". 


\author{
- Tec.Enf05: (...) “Encaminhar o paciente para Unidade de Recuperação Pós-anestésica \\ (URPA)".
}

- Enf01: (...) "Monitoriza para verificação de dados vitais (Pressão arterial, Frequência cardíaca, saturação de oxigênio). Atentar para o nível de consciência. Acompanhar as queixas do momento. Verificar função renal, com apresentação da diurese".

- Tec.Enf01: (...) "Realizar medicações de acordo com prescrição médica. Observar sinais de hemorragia após o procedimento, presença de hematoma ou sangramento".

- Enf01: (...) "Orientações: Repouso com o local do procedimento, caso seja radial 4 horas, femoral 6 horas. Comunicar enfermagem caso sinta alguma coisa".

O pós-operatório é o momento que visa à reabilitação do doente e o fornecimento de orientações para a alta hospitalar (MONTEIRO EL et al., 2014).

O estudo publicado por Regis AP (2017) o processo de enfermagem torna-se relevante para a realização do cuidado, pois implica o conhecimento na orientação, resultando no entendimento das ações planejadas. Entretanto, para que seja aplicado de forma adequada, o enfermeiro deve conhecer os procedimentos, os benefícios, riscos e suas complicações para conferir a intervenção.

Mediante isso, a aplicação do processo de enfermagem torna-se importante na assistência prestada ao paciente submetido à angioplastia coronária, como afirma os participantes do estudo.

- TEnf08: (...) "A importância para o paciente é desde o início que ele chega ao hospital porque eu tenho a padronização de uma assistência qualificada, o paciente é bem cuidado e pode evoluir para uma melhora mais rápido".

- Enf01: (...) "Porque é um cuidado diferenciado com o paciente, né, então analisamos o paciente como um todo, é os riscos que ele está exposto, é os diagnósticos, é verifica a necessidade para aquela assistência do paciente".

- TEnf04: (...) "A importância é que você tenha um cuidado mais adequado com esse paciente de forma total, de forma é sistematizada, porque você vai pegar seu paciente desde o início, desde a primeira abordagem, e vai acompanhá-lo até a liberação, até a alta desse paciente, então, é total fundamental importância para que você conheça e saiba lidar com as características especificas de cada um".

Focando na aplicação do processo de enfermagem, deve-se desenvolver, organizar e uniformizar a assistência para os pacientes submetidos à angioplastia transluminal percutânea coronária para que sejam valorizados individualmente e assistidos de forma integral.

- TEnf02: (...) "É muito importante você cuidar do paciente como um todo, né, então, a equipe de enfermagem tem que trabalhar todos juntos, é importante porque assim podemos prestar um serviço de qualidade, pois somos acreditados em nível III, e isso confere ao nosso paciente segurança e qualidade, é muito importante para que ele saia daqui satisfeito e para que o nosso serviço tenha sido um serviço de excelência".

- Enf02: (...) É muito importante porque ela vai focar aquilo que é mais necessário para assistência do paciente. Afinal de contas o que a gente trabalha aqui é uma qualidade melhor de saúde para o paciente, né.

O papel da equipe de enfermagem frente à aplicação do processo de enfermagem é de extrema importância, pois as intervenções tornam-se mais eficazes para redução de complicações e diminuição de riscos dos pacientes. Também, atendendo a todos que estão à volta deste, seus familiares e acompanhantes. 


\section{Orientação para alta hospitalar}

A orientação adequada evita o despreparo do paciente, tornando-se imprescindível conhecer as bases teóricas para desenvolver o cuidado com intuito de proporcionar uma reflexão sobre a relação enfermeiro/paciente de modo a desvendar e propor novas maneiras de cuidado (AMESTOY SC et al., 2009). Permite ainda que o paciente tenha uma reação positiva aos cuidados que necessitará prestar após alta hospitalar, possibilitando recuperação efetiva.

O enfermeiro, além de atuar no atendimento ao paciente, também deve fornecer orientação, focando na prevenção dos fatores de risco e nas informações quanto ao reconhecimento dos sintomas do IAM; deve também enfatizar a importância do atendimento imediato a partir do surgimento do desconforto torácico (SAMPAIO ES e MUSSI FC, 2009).

Estas orientações devem contemplar o preparo físico, o procedimento e os cuidados a serem realizados. Desse modo, as orientações visam evitar complicações decorrentes do pós- procedimento e proporcionar uma melhora rápida ao paciente (LACCHINI AJB et al, 2011).

Diante disso, a alta hospitalar significa a transferência da responsabilidade da continuação do tratamento para o paciente e para a família. Portanto, a equipe de enfermagem torna-se primordial na orientação e no preparo do paciente, para assumirem responsabilidades no plano terapêutico, de forma que suas necessidades sejam atendidas, evitando complicações futuras.

Ao questionar a equipe de enfermagem sobre as orientações para alta hospitalar, foram destacadas as seguintes orientações como primordiais:

- Enf01: (...) "Se tiver alguma alteração no local da punção, retornar para o hospital para avaliação".

- Tec.Enf06: (...) "No mínimo repouso três dias, sem realizar qualquer atividade, tipo dirigir, caminhar, correr, andar de bicicleta".

- Enf02: (...) "Procurar o centro de saúde mais próximo, no caso de hemorragia em casa, comprimir o local com ajuda de alguém e encaminhar para o médico". (...) "Pacientes diabéticos que suspendeu a medicação, retornar no dia após o exame".

- Tec.Enf07: (...) "Retirada desse curativo após 24h, em casa mesmo, no posto de saúde e a questão do retorno ao cardiologista se houver necessidade".

- Tec.Enf04: (...) "Estar agendando uma consulta com o médico pra poder verificar a questão das medicações que ele tá tomando e levar o resultado do exame para o médico".

-Tec.Enf03 (...) "Manter uma alimentação adequada".

A orientação ao paciente e à família para a alta hospitalar é um fator de extrema importância para a recuperação no ambiente extra-hospitalar. $O$ bom desempenho permite mudanças de comportamento em curto ou em longo prazo e possibilita ao paciente expressar preocupações e dúvidas quanto aos problemas relacionados à saúde. O estudo realizado apresentou algumas limitações importantes por ser desenvolvido apenas em 02 hospitais, com os profissionais de enfermagem que explanaram os seus conhecimentos teórico-práticos para o processo de enfermagem, no qual o número de colaboradores é diferenciado e reduzido. Outro fator relevante é a falta da literatura científica na área, limitando a explanação sobre o processo de enfermagem no serviço de hemodinâmica.

\section{CONCLUSÃO}

Diante dos achados, percebe se que os enfermeiros demonstram satisfação em trabalhar no serviço de hemodinâmica, mas apontam dificuldades no serviço como: manter a rastreabilidade do material em dia, relacionamento interpessoal e liderança com a equipe. Contudo, evidencia nesse estudo, a relevância do 
processo de enfermagem na atuação profissional em pacientes submetidos à angioplastia coronária, como um instrumento capaz de produzir mudanças no trabalho da enfermagem e melhorar a qualidade da atenção/assistência prestada ao indivíduo e família. Por fim, a pesquisa poderá contribuir como instrumento de orientação do trabalho da equipe de enfermagem em serviços de hemodinâmicas.

\section{REFERÊNCIAS}

1. AMESTOY SC, et al. As percepções dos enfermeiros acerca da liderança. Rev Gaúcha Enferm. 2009; 4(30):61724.

2. BARBOSA MHM, et al. Complicações em pacientes submetidos a angioplastia coronária transluminal percutânea. Rev. Eletrônica trimestral de Enfermeria. Uberaba. 2013; (31):24-33.

3. BRASIL.2016. Organização Pan-Americana da Saúde. Doenças cardiovasculares . Disponível em <http://www.paho.org/bra/index.php> Acesso em: 04. Out. 2017

4. BRASIL. 2017. Organização Pan-Americana da Saúde. Doenças cardiovasculares. Disponivel em: <http://www.paho.org/bra/index.php> Acesso em: 04. Jan. 2018

5. DELLÍACQUA MCQ, MIYADAHIRA AMK. Ensino do processo de enfermagem nas escolas de graduação em enfermagem do estado de São Paulo. Rev Latino-am Enfermagem 2002;10(2):185-91

6. LACCHINI AJB, et al. Importância das orientações do enfermeiro para pacientes no período pré-operatório. Revista Contexto \& Saúde. 2011; 10(20):1021-1024

7. LEMOS IMN, et al. O papel do enfermeiro dentro da unidade de hemodinâmica. International nursing congress Theme: Good practices of nursing representations In the construction of society. 2017; 9-12.

8. LIMA LR, et al. Nursing diagnoses in patients post-angioplasty transluminal percutaneous coronary based on the horta's assumption. Rev enferm UFPE on line. 2008; 2(3):205-12

9. LINCH GFC, et al. Enfermeiros de unidades de hemodinâmica do Rio Grande do Sul: perfil e satisfação profissional. Texto Contexto Enferm. 2010; 19(3):488-95.

10. MARCEDO VL. Validação de conteúdo das intervenções de enfermagem "Ensino: Pré-operatório" e "Ensino: Procedimento/Tratamento" da Classificação das Intervenções de Enfermagem, para procedimento percutâneo em hemodinâmica. SP. Dissertação (Mestrado em Enfermagem) - Universidade Estadual Paulista "Júlio de Mesquita Filho". Faculdade de Medicina, Botucatu. 2016; 99p.

11. MONTEIRO EL, et al. Cirurgias seguras: elaboração de um instrumento de enfermagem perioperatória. Rev. SOBECC, São Paulo. 2014;19(2): 99-109

12. NOGUEIRA JA. Gestão da doença crônica com o desafio dos custos. Revista Saúde, 2004; (2).

13. OLIVEIRA MF, SILVA LF. Enfermagem em laboratório de hemodinâmica: diagnóstico e intervenção fundamentados na Teoria da Adaptação de Roy ${ }^{1}$. Rev. Eletr. Enf.2010; 12(4), 678-85.

14. PINHO MCG.Trabalho em Equipe de Saúde: limites e possibilidades de atuação eficaz. Revista Ciência e Cognição. 2006; (8)

15. REGIS AP, et al. Cuidados de enfermagem no cateterismo cardíaco e angioplastia coronariana: desenvolvimento de um instrumento. Revista Recien. 2017; 21(7):3-20

16. SAMPAIO ES, MUSSI FC. Cuidado de enfermagem: evitando o retardo pré-hospitalar face ao infarto agudo do miocárdio / Cuidado de enfermería: evitando el retraso prehospitalario frente al infarto agudo del miocardio / Nursing care: reducing pre-hospitalization delay in acute myocardial infarction. Rev. enferm. UERJ. 2009; 3(17):442-446.

17. SANTOS WN. Sistematização da Assistência de Enfermagem: o contexto histórico, o processo e obstáculos da implantação. Journal of Management \& Primary Health Care. 2014;5(2):153-158.

18. SILVA MLC. A importância da consulta de enfermagem para o cliente no pré-operatório de cirurgias eletivas. Monografia (Especialização em Linhas de Cuidado em Enfermagem) - Departamento de Enfermagem da Universidade Federal de Santa Catarina. 2017; 16p.

19. TAETS GGC. Cuidados de enfermagem e diagnósticos para pacientes submetido à angioplastia coronária transluminal percutânea. Revista Recien. São Paulo. 2016; 16(6):3-10.

20. VIEIRA LC, et al. Dificuldades e Necessidades da Equipe de Enfermagem em Serviços de Hemodinâmica e Angiografia. Arq Ciênc Saúde. 2009; 1(16):21-5. 